\title{
Indústria 4.0: O Papel das Federações Empresariais
}

Industry 4.0: The Role of Industry Federations

\section{Resumo}

A adoção das tecnologias digitais que fizeram emergir a $4^{a}$ Revolução Industrial tornou-se crítica no ambiente de negócios da economia pós-industrial, trazendo para economias emergentes pressões ainda maiores no processo de globalização de suas empresas. $\bigcirc$ objetivo principal deste artigo foi entender como, na percepção dos empresários, a estratégia do Sistema FIRJAN pode colaborar para o desenvolvimento de políticas de estímulo à inovação e à inserção das empresas fluminenses na Indústria 4.0. $\bigcirc$ estudo adota uma abordagem qualitativa com finalidade exploratória, as evidências coletadas foram tratadas por um processo de codificação auxiliado pelo software ATLAS.ti. Os resultados obtidos indicaram que embora a rede de institutos de inovação contribuísse para a inovação localizada, ficou evidente a falta de políticas orientadas para inovação que promovam a real transformação da indústria fluminense. As narrativas obtidas indicam que grande parte dos empresários considera as tecnologias digitais ou virtuais um luxo dispensável. Esta resistência fortalece a percepção de que a indústria 4.0 é considerada uma realidade muito distante. De modo a superar uma visão míope e autocentrada de segredo industrial, a FIRJAN, deve criar um senso de urgência nos empresários e estimular, ainda que indiretamente, a troca de conhecimentos tecnológicos entre as empresas do estado.

\section{Abstract}

The adoption of the digital technologies that gave rise to the 4 th Industrial Revolution became critical in the business environment of the post-industrial economy, bringing to the emerging economies even greater pressures in the process of globalization of their companies. The main objective of this article was to understand how, in the perception of business executives; the strategy of the FIRJAN System could contribute to the development of policies to stimulate innovation and the insertion of Rio de Janeiro companies in the 4th Industrial Revolution. The study adopts a qualitative approach with exploratory purpose and the evidences collected were treated by a coding process aided by ATLAS.ti software. The results indicate that although the network of innovation institutes contributed to localized innovation, it was evident the lack of innovation-oriented policies that promote the real transformation of the industry of Rio de Janeiro. The narratives obtained indicate that most business executive consider digital or virtual technologies an expendable luxury. This resistance strengthens the perception that industry 4.0 is considered a very distant reality. in order to overcome a myopic and self-centered vision of industrial secrecy, FIRJAN can stimulate, albeit indirectly, to the exchange of technological knowledge among companies.
Palavras Chave: Sistema Nacional de Inovação. Estratégias Operacionais. Indústria 4.0 .Política Tecnológica. Representação Empresarial.
Keywords: National Innovation System. Opera tional Strategies. Industry 4.0. Technology Policy. Business Representation. 


\section{INTRODUÇÃO}

A difusão das tecnologias digitais alterou profundamente as estratégias operacionais, colocando a competição global em um novo patamar. A adoção das tecnologias digitais que fizeram emergir a $4^{\mathrm{a}}$ Revolução Industrial tornou-se crítica no ambiente de negócios da economia pós-industrial, trazendo para as economias emergentes pressões ainda maiores no processo de globalização de suas empresas. Por conseguinte, a adoção de ações concretas para o fortalecimento de um ecossistema de inovação fundamentado no cooperativismo e na ação conjunta dos setores empresarial e público, capaz de ir além da simples sobrevivência (Mazzucato, 2015), será cada vez mais importante, particularmente no que tange à inovação tecnológica.

Na tradição de políticas de estímulo à industrialização, o conceito de Sistema Nacional de Inovação (SNI), desenvolvido a partir da primeira metade da década de 1990 por economistas ligados à escola neoschumpeteriana (Nelson, 1993), destaca a ação das diferentes instituições que influenciam na capacidade inovativa de um país. Entretanto, nos estudos científicos que usam a lente teórica do SNI não se discute o papel das entidades cooperativas empresarias. Sendo assim, este artigo contribui para a teoria e prática de administração na medida em que busca investigar como tende a suceder o processo de mudança, o papel das organizações empresariais e suas organizações cooperativas no enfrentamento das oportunidades e ameaças decorrentes da $4^{\text {a }}$ Revolução Industrial.

O objetivo principal deste artigo é entender como, na percepção dos empresários, a estratégia do Sistema FIRJAN pode colaborar para o desenvolvimento de políticas de estímulo à inovação e à inserção das empresas fluminenses na Indústria 4.0.

Com vistas a melhor atender o objetivo principal, este artigo buscará responder aos seguintes objetivos secundários: i) Explorar os impactos da Indústria 4.0 para a economia brasileira; ii) Entender como a atuação das entidades empresariais na defesa de interesses contribui para a evolução do Sistema Nacional de Inovação; iii) Comparar a percepção dos empresários com a percepção dos executivos a respeito das estratégias adotadas pelo Sistema FIRJAN para a Indústria 4.0; iv) Identificar as condições necessárias para a contínua absorção de tecnologias digitais, tanto em produtos quanto em processos, na busca da melhoria dos padrões de desempenho nas organizações e na construção de capacidades tecnológicas.

Esta pesquisa é relevante na medida em que o PIB industrial do Rio de Janeiro em 2015 representa $11,3 \%$ da indústria nacional. As empresas industriais em 2016 no estado representam 5,1\% do total de empresas que atuam no setor industrial do Brasil. O Sistema FIRJAN é composto por cinco organizações SESI; SENAI; IEL que reúnem e oferecem soluções de educação e inovação para a indústria fluminense, além da FIRJAN e do CIRJ. Representante legal de 103 sindicatos patronais industriais, a FIRJAN atua nas esferas municipal, estadual e federal para defender questões que impactam diretamente a competitividade da indústria. 
A contribuição teórica deste artigo está na combinação da triangulação dos conceitos de SNI, do sistema corporativista de representação de interesses empresarias, e estratégias operacionais no contexto da $4^{\mathrm{a}}$ Revolução Industrial.

O estudo contempla 05 sessões, sendo que na primeira sessão temos a introdução com parágrafos por onde passa a ideia de pesquisa, o objetivo com o trabalho e a pergunta de pesquisa; na segunda sessão encontramos o referencial teórico que nos ajudará a substanciar o problema de pesquisa e relacionar o presente estudo ao diálogo corrente na literatura que vem se desenvolvendo; na terceira sessão temos a metodologia e o como foi desenvolvida a pesquisa; os resultados encontrados estão na quarta sessão. Por fim, são apresentadas as conclusões e outras considerações sobre o tema e a pesquisa na quinta sessão.

\section{REFERENCIAL TEÓRICO}

\subsection{Sistema Nacional de Inovação (SNI)}

Segundo Freeman (1995), o SNI é um conjunto de instituições, atores e mecanismos em um país que contribuem para a criação, avanço e difusão das inovações tecnológicas. Para Resele (2015), o SNI destaca a cooperação entre o setor público e privado para criar, acumular, mudar e usar novos conhecimentos com o objetivo de promover a implantação da inovação e, subsequentemente, o desenvolvimento sustentável da economia.

A abordagem de sistemas de inovação (SI) permite compreender e orientar os processos de criação, uso e difusão do conhecimento, está relacionado ao interesse em compreender as mudanças técnicas e as trajetórias históricas e nacionais rumo ao desenvolvimento (CASSIOLATO; LASTRES, 2005).

O SNI brasileiro é caracterizado pela imaturidade institucional, com o predomínio de instituições públicas marcadamente burocráticas, estrutura de mercado frágil, instrumentos regulatórios não estabelecidos e políticas de estímulo à competitividade industrial ineficaz (MAMEDE et al., 2016).

\subsection{O Sistema Cooperativo das Federações Industriais}

O sistema corporativista de representação de interesses foi instituído no Brasil pela legislação sindical da década de 1930, sob o governo de Getúlio Vargas (MANCUSO, 2007). Sucedeu da necessidade de sindicalização da classe e das vantagens em unificar a força da indústria, tendo como objetivo principal a coordenação, proteção e representação legal das diversas categorias econômicas da indústria, e a promoção da defesa de seus legítimos interesses (BRAGA, 2013). Doctor (2002) aborda o termo "sistema corporativista de representação de interesses" como sendo um tipo de seto- 
rização corporativa, onde o corporativismo refere-se à estrutura formal de representação de interesses imposta pelo Estado, utilizada para incorporar os grupos emergentes do incipiente processo de industrialização e modernização.

Criado há mais de 70 anos, o sistema corporativista subsiste até hoje, tendo passado por modificações importantes, no entanto, sem ter perdido seus traços fundamentais. A Confederação Nacional da Indústria (CNI) é a entidade de cúpula do sistema corporativista de representação dos interesses da indústria (Mancuso, 2007).

\subsection{A Indústria 4.0}

A inovação tem influência positiva determinante para a competitividade das empresas e o desenvolvimento econômico dos países (FREEMAN; SOETE, 1997; PINSKY et al., 2015). As inovações sistêmicas, ao contrario das inovações incrementais, ao romperem com a rota tecnológica dominante seguem um longo processo de difusão até que as demais tecnologias à montante e à jusante na cadeia produtiva se consolidem (DOSI, 1982).

A Indústria 4.0, considerada neste artigo como uma inovação tecnológica sistêmica, decorre da introdução de diferentes tecnologias digitais, tais como Internet das Coisas (IoT), computação nas nuvens, Big Data e inteligência artificial, entre outras, para que os processos de negócios e de engenharia estejam profundamente integrados, de modo que a produção funcione de forma flexível, eficiente e verde, com alta qualidade e baixo custo (WANG et al., 2015). A introdução do protocolo de Internet IPv62 em 2012 impulsionou o fenômeno batizado como "Internet das Coisas" (IoT), permitindo que microcomputadores autônomos conectados em rede possibilitem a convergência entre os mundos físico e o virtual por meio de “Cyber-Physical Systems” (CPS) ou Sistemas Ciber-Físicos (KAGERMANN et al., 2013).

Definido como uma tecnologia transformadora para a gestão do grande volume de dados gerados, também conhecido como Big Data, o Sistemas Ciber-Físicos (CPS) tem a finalidade de alavancar a interconectividade das máquinas para o alcance do objetivo de máquinas inteligentes, resilientes e auto adaptáveis. O conhecimento agregado de componentes e informações no nível de máquina fornece auto-configuração e auto-manutenção para a fábrica que garante uma produção livre de problemas e também oferece planejamento de produção otimizado para gerenciamento da fábrica (LEE et al., 2014).

O desenvolvimento de sistemas de produção baseados em tecnologias da informação e comunicação (TIC) com tecnologias de fabricação de alta qualidade e capacidades inteligentes são fundamentais para a indústria na era 4.0, pois visam aperfeiçoar seu desempenho com um alto grau de autonomia e adaptabilidade para uma combinação equilibrada de alta produtividade com produção precisa, incluindo a implementação do conceito de manufatura sustentável (JARDIM-GONÇALVES 
et al., 2016).

O termo Indústria 4.0 surge a partir de uma "iniciativa estratégica" do governo alemão adotada como parte do Plano de Ação de Estratégia de Alta Tecnologia em novembro de 2011 (WANG et al., 2015). Assim como em Kagermann et al., (2013) a pesquisa documental indicou as seguintes iniciativas dos SNI:

(i) EUA: O Programa de Pesquisa e Desenvolvimento de Tecnologia da Informação (NITRD, 2017) que reúne 19 agências para coordenação de pesquisa em diferentes domínios de TI, incluindo interação homem-máquina e gerenciamento de informação. O setor privado lidera o Advanced Manufacturing Partnership (AMP, 2017) para traçar um curso ao investimento e promoção do desenvolvimento de tecnologias emergentes, facilitar a criação de redes entre iniciativas governamentais, universitárias e privadas neste campo;

(ii) CHINA: onde o Centro IoT (IMECAS, 2017) investiga tecnologias básicas IoT e requisitos de padronização associados, a "Zona de inovação IoT" na cidade de Wuxi, que reúne cerca 300 empresas e mais de 70 mil pessoas;

(iii) EUROPA: investimentos bilionários chamam a atenção para promoção de P\&D na área da fabricação inteligente orientados para TIC, além da formulação de documento para discussão sobre financiamento de futuras pesquisas e iniciativas se desenvolvem, vide: o projeto "IoT@Work" da Siemens, a plataforma tecnológica (ARTEMIS, 2017);

(iv) ÍNDIA: O Centro de Inovação de Sistemas Ciber-Físicos lançado para realizar pesquisas em uma variedade de áreas, incluindo a robótica humanoide (IITH, 2017).

\section{METODOLOGIA}

A pesquisa parte de uma perspectiva epistemológica construtivista e com uma abordagem qualitativa. Optou-se por um estudo exploratório, uma vez que "ainda não foi escrito muita coisa sobre o tópico, o pesquisador tentará ouvir os participantes e construir um entendimento baseado nas ideias deles" (CRESWELL, 2007, p.46), e também reflete uma conjunção inequívoca com o objetivo deste estudo. Os dados empíricos foram coletados por meio de uma abordagem métodos múltiplos, interativos e humanísticos. A coleta combinou três etapas distintas: observação participante, entrevistas semiestruturadas e análise de documentos que incluem um vasto leque de materiais. As entrevistas semiestruturadas (roteiro de entrevista) foram feitas cara-a-cara com os executivos responsáveis pelas 05 principais áreas corporativas do Sistema FIRJAN, conforme consta na Tabela 1, e também com empresários de pequenas e médias empresas, fundadas no estado do Rio de Janeiro, e residentes em cidades distintas da capital conforme consta na Tabela 2. Por motivo de confidencialidade, nomes de pessoas e empresas não serão publicados. 
Tabela 1 - Perfil dos executivos entrevistados

\begin{tabular}{c|ccc}
\hline Entrevistado & Área de atuação & Formação & Anos de empresa \\
\hline Executivo SF 1 & Educação Corporativa & Especialização & 29 \\
Executivo SF 2 & Estratégia de Inovação & Mestrado & 15 \\
Executivo SF 3 & Estratégia de Mercado & Especialização & 13 \\
Executivo SF 4 & Estratégia Tecrológica & Especialização & 7 \\
Executivo SF 5 & Planej. Estratégico & Mestrado & 20 \\
\hline
\end{tabular}

Fonte: Desenvolvido pelos autores.

Tabela 2 - Perfil dos empresários entrevistados

\begin{tabular}{|c|c|c|c|c|c|c|}
\hline Indústria & Setor & Porte & $\begin{array}{l}\text { Área de } \\
\text { atuação }\end{array}$ & Fundação & Entrevistado & $\begin{array}{l}\text { Anos de } \\
\text { empresa }\end{array}$ \\
\hline Empresário 1 & M. Mecânico & Médio & Continental & 1957 & Diretor de Oper. & 20 \\
\hline Empresário 2 & Alim. Bebidas & Pequeno & Estadual & 2002 & Gerente Geral & 3 \\
\hline Empresário 3 & Ed. Gráfica & Médio & Nacioral & 1901 & $\begin{array}{l}\text { Ass. Jurídico } \\
\text { / Pres. Sindicato }\end{array}$ & 29 \\
\hline Enipresário 4 & M. Mecânico & Grande & Mundial & 1951 & Engenheiro & 9 \\
\hline Empresário 5 & $\begin{array}{l}\text { Alim Bebidas } \\
\text { Extrativista } \\
\text { Construção } \\
\text { Metalurgia }\end{array}$ & Grande & Estadual & 1999 & $\begin{array}{l}\text { Diretor Oper. / } \\
\text { Pres. Regional } \\
\text { FIRJAN }\end{array}$ & 18 \\
\hline Eripresário 6 & $\begin{array}{l}\text { Alimi e } \\
\text { Bebidas }\end{array}$ & Pequeno & Estadual & 2008 & Diretor Oper. & 9 \\
\hline Empresário 7 & Moda & Pequeno & Estadual & 2005 & Diretor Oper. & 12 \\
\hline
\end{tabular}

Fonte: Desenvolvido pelos autores.

As entrevistas com os especialistas foram realizadas no mês de outubro/2017 e tiveram duração de aproximadamente 1 hora. Foram gravadas e transcritas de forma manual a fim de reter os elementos transcorridos, incluindo as interações não verbais e sociais. Para a análise documental recorremos a documentos internos da empresa, com o recurso a várias fontes de dados para a coleta de dados, tais como: Mapa do Desenvolvimento de Estado do Rio de Janeiro 2016-2025; Relatório anual de inovação SENAI 2016; Cadernos SENAI de inovação; Revista Informe Inovação - FIRJAN; o que 
possibilitou a triangulação das informações. As transcrições das entrevistas foram transferidas para o ATLAS.ti e codificadas. A codificação dos dados ocorreu a partir de categorias encontradas na literatura de Souza (2016), Resele (2015) e a partir da fala dos próprios entrevistados. Foram analisadas as relações entre os códigos com objetivos de responder as perguntas de pesquisa, identificar novas questões e padrões, juntar as ideias e integrar as descobertas coletadas.

\subsection{A Estratégia do Sistema FIRJAN}

Uma alternativa criada pela federação para a obtenção de um objetivo comum que beneficie a indústria do Rio de Janeiro está configurada no Mapa do Desenvolvimento do Estado do Rio de Janeiro (2016-2025). A inovação da indústria do estado do Rio de Janeiro esta posta como um assunto secundário dentro do todo contexto deste documento. Alocado no pilar "Gestão Empresarial” e devido à necessidade de manter o foco em relação ao objetivo da pesquisa, destacaremos apenas o objetivo 3 que propõe "Promover a inovação e o desenvolvimento tecnológico" com ações relacionadas abaixo:

- Fortalecimento do ambiente de inovação através da alteração do marco legal da inovação visando maior incentivo às empresas para o desenvolvimento de PD\&I e o fortalecimento da gestão da inovação; da ampliação da oferta de serviços tecnológicos no estado; da oferta de cursos e serviços de gestão da inovação às empresas associadas; da ampliação da cooperação entre empresas e integração com os demais atores de inovação; da identificação e estímulo ao desenvolvimento de empresas de base tecnológica, voltadas para a indústria do estado e; da disseminação da utilização da propriedade intelectual como fonte de informação tecnológica e vantagem competitiva.

- Fomento a inovação e o empreendedorismo com estímulo a participação de startups em missões e fóruns de inovação nacionais e internacionais; com a promoção de encontros periódicos para atração de startups e aproximação com atores de acesso a mercados, investidores, habitats de inovação, grandes empresas e aceleradoras; por meio do fortalecimento da capacidade das empresas fluminenses para acessar, absorver e desenvolver inovações tecnológicas e não tecnológicas; e da disseminação de informações sobre novas fontes de financiamento, acordos internacionais, tecnologias e inovações tecnológicas emergentes nacional e internacionalmente que tenham potencial impacto para a indústria.

- Acesso a recursos financeiros para a inovação no estado e apoio as empresas associadas em projetos de investimentos, novos negócios e startups. Acesso a recursos financeiros para a inovação no estado e apoio as empresas associadas em projetos de investimentos, novos negócios e startups e; ampliação do acesso a fontes alternativas de garantias para empréstimos, tais como fiança bancária, fundos garantidores e seguro-garantia. 


\title{
4. RESULTADO E DISCUSSÃO
}

Com uma atuação verticalizada, a federação objetiva impactar um número expressivo de empresas. Ao tratar questões com as lideranças dos sindicatos patronais que representam uma região, entende que a defesa de interesse está relacionada às demandas especificas do setor de atuação de seus filiados.

A realidade do discurso em prol de uma nova era industrial no estado do Rio de Janeiro contrasta com o longo caminho a ser percorrido, uma vez que se trata de um tema novo para os atores locais. Setorialmente, o Sistema FIRJAN tem procurado identificar o nível tecnológico de indústria regional, e ponderando a respeito de uma possível implantação inicial da indústria 4.0. O primeiro ponto a ser superado é levar para o empresário o que esse novo paradigma representa e o Sistema FIRJAN tem se movido no sentido de disseminar o conhecimento e fundamentalmente na capacitação do empresário neste momento onde ele tem que tomar decisões estratégicas em meio ao grande volume de dados e informações disponíveis que adentram as empresas.

A gestão tecnológica regional traz a reboque algumas questões agravantes para a situação da inovação na região. A análise revelou uma forte densidade relacionada à contribuição para que a empresa, a partir do Sistema FIRJAN, integre e desenvolva novas tecnologias e conhecimentos aplicáveis em sua área, gerando inovação e melhorias de seus processos. De certa forma, este fator tende a estar relacionado ao grau de maturidade da gestão dos empresários brasileiros, da região.

“O ponto principal é que eu vejo que poucas pessoas realmente gostam do seu negócio a ponto de querer investir para que aquilo tenha futuro. Eu vejo muito empresário querendo sugar o que o negócio dá. Não destina uma verba para essa inovação. E no momento que a gente está vivendo de crise se a pessoa nunca fez isso, não é agora que vai fazer. Que o faturamento reduziu em todos os seguimentos praticamente. Então vejo certa resistência mais por conta disso. As pessoas se interessam, acham legal, mas eu vejo poucas pessoas dispostas a pagar pela inovação". (Empresário 6)

O “dia a dia” do negócio limita a visão do empresariado.

\begin{abstract}
"A maior parte das empresas do Rio de Janeiro são pequenas e médias e sempre, sempre não, mas assim, em geral as pequenas e médias, tem mais dificuldades de inovar. A liderança, ela está envolvida em tudo, ela está envolvida em todas as fases da pequena empresa e ai parar para pensar naquilo (estratégia e inovação), você é engolido pelo dia a dia, engolido por questões operacionais, então você não para muito para pensar naquilo (estratégia e inovação)". (Executivo SF 5).
\end{abstract}

O desafio maior está em transpor a fronteira da capacitação, a federação se aproximar ainda mais da indústria de alguma forma questionando, vendo onde ela pode apoiar a absorção dessas tecnologias. 
"Atuar mais nessas fases de disseminação e de apoio na absorção dessas tecnologias, que eu acho que a gente oferece e vende, mas a gente não provoca a gente não fala para o cara que aquilo é importante, a gente não ajuda ele a fazer um diagnóstico para o negócio dele, o quanto ele deve implantar aquilo ou não". (Executivo SF 5)

"A FIRJAN pode fazer ainda mais do que faz, é acompanhar o todo. Ah vocês foram lá e ai houve algum resultado? Vocês conseguiram fazer o processo? Teve algum problema no meio do caminho? A gente pode apoiar de alguma forma? Então acho que falta, ela da uma orientação inicial, mas talvez não acompanhe o todo". (Empresário 1).

Observamos que a federação relaciona o governo como ator fundamental para o ecossistema de inovação, ao "puxar a bandeira", para fortalecer as convicções por meio de uma política estruturada para o desenvolvimento da indústria. Porém, a falta de políticas orientadas para inovação se apresenta na pesquisa como uma das principais barreiras para a atuação do Sistema FIRJAN no processo inovativo

"A gente sofre de não ter políticas orientadas para inovação e que transformem o país e mantenham-se estáveis minimamente durante processos de crise. No Brasil, a gente tem uma visão muito errada, no momento em que a gente entra numa crise, normalmente as empresas, o estado e o país, eles cortam os investimentos em inovação, tecnologia e inovação. Então, acaba que a gente cria um buraco muito maior do que do que a gente já tem" (Executivo SF 2).

Em relação à questão de políticas públicas, se observa a necessidade de atuação propositiva a partir dos interesses conjuntos, suprimindo assim o sentimento de uma realidade distante para a evolução da indústria.

"Brasil, nossa realidade, e ai as dificuldades das federações, pouco se pode fazer a não ser provocar, a gente tem uma área de defesa interesse que está ai brigando não só por tecnologia, mas todos os assuntos que dizem respeito ao desenvolvimento da indústria". (Executivo SF 4).

A importância dada ao nível de tecnologia deriva do tipo de indústria atual e dos caminhos que escolherá para si.

"A gente está falando de indústria 4.0, a gente tem que considerar que $60 \%$ a $70 \%$ do parque fabril aqui, principalmente aqui no Rio de Janeiro, são máquinas extremamente antigas, são máquinas obsoletas, são máquinas de 1970 de 1980, que não tem automação nenhuma, a gente ainda está falando de ter um kit que faça uma automação XYZ em um torno muito antigo. Para você gerar alguma expectativa de ter algum trabalho mais automatizado, de ter uma reprodutibilidade, repetitividade de uma atividade. Mas cara, a gente ainda tem um parque muito atrasado, que requer ainda na indústria 4.0, que as máquinas elas sejam máquinas novas, atualizadas que tenham sistemas embarcados, que tenha conteúdo, que tenha acesso, você não tem, o empresário não vai trocar a fábrica dele inteirinha por uma máquina nova". (Executivo SF 4).

Sob a perspectiva da nova dinâmica do mercado que está se formando, a federação reconhece uma nova realidade onde o conhecimento, a competitividade e a conectividade, acenam para oportunidades que abarcam a necessidade de adaptação do comportamento para atender aos requisitos da nova revolução industrial, a cultura e a educação. 
"O grande benefício para a sociedade é a forma como a gente vai usar esse serviço, e ai falando de serviço, para nós brasileiros, a gente vai conseguir se beneficiar dessa indústria mais pela parte de Big Data, de conteúdo, de Analitics. Estou olhando muito pouco benefício para o Brasil, para a sociedade brasileira de médio prazo, de curto médio prazo em termos de machine learning, inteligência artificial, tirando o robozinho que se cria para extrair um dado, não consigo imaginar ainda a gente tendo um carro autônomo no meio da rua, cidade inteligente, aquelas que você vai ter um trânsito que consiga controlar o fluxo de acordo com o tempo, com o volume de carros. Você vai ter partes disso, de um Wearable [...], são essas coisas que a gente vai se beneficiar". (Executivo S

\section{CONSIDERAÇÕES CONCLUSIVAS}

Os documentos investigados na pesquisa indicam que o Sistema FIRJAN entendia que exercia um papel importante no apoio às empresas, tanto como representação de interesses, e como agente de promoção da indústria. Segundo a federação, a lógica de atuação setorial, que estava sendo implantada na época desta pesquisa, permitia informar às empresas as principais tendências do mercado e avançar a educação para a formação de técnicos que pratiquem a inovação dentro das indústrias. A estrutura de rede de institutos de tecnologia e inovação, demostrava que o Sistema FIRJAN realizava pesquisa aplicada na resolução de problemas pontuais. Na realidade regional, as evidências coletadas indicam poucos empresários dispostos a se arriscar pelo caminho da inovação de caráter radical. Culturalmente envolvidos em todas as fases do processo produtivo, os empresários demonstraram estar engolidos pelo dia a dia das questões operacionais e possuir um limitado alcance dos impactos estratégicos da $4^{a}$ Revolução Industrial, embora a Firjan indicasse em seus documentos o caráter disruptivo desta tecnologia. O papel passivo ou reativo em termos de competividade se transforma em uma grande barreira para a renovação do parque industrial do Rio de Janeiro formado em sua maioria por pequenas e médias empresas.

Não identificamos na fala dos entrevistados a preocupação como aquela encontrada na pesquisa documental, nas quais as ações estratégicas do estado e dos empresários evidenciam um esforço no sentido de expandir a capacidade tecnológica por meio das tecnologias digitais, estabelecendo objetivos para reduzir a dependência da tecnologia estrangeira e para obter a liderança tecnológica global, incluindo a fabricação de equipamentos e a tecnologia de informação de ponta. Diferentes disto, os resultados corroboram para uma percepção, no que diz respeito à inovação disruptiva, o SNI brasileiro é caracterizado pela imaturidade institucional, com o predomínio de instituições públicas marcadamente burocráticas, estrutura de mercado frágil, instrumentos regulatórios não estabelecidos e políticas de estímulo à competitividade industrial ineficaz.

Por não se tratar de um programa específico para trabalhar a inovação, o Mapa do Desenvolvimento do Estado do Rio de Janeiro (2016-2025) não se mostrou capaz de protagonizar a real transformação da indústria. Sendo assim, os fatores agravantes na atuação do Sistema FIRJAN em apoio à inovação tecnológica tendem a se repetir, ou mesmo aumentar com o advento da $4^{\mathrm{a}}$ Revolução Indus- 
trial. Por conseguinte, torna-se urgente à adoção de iniciativas que formulem estratégias concretas de inovação e roteiros de implementação destinada a tornar a indústria do estado do Rio de Janeiro fornecedor de soluções que reduzam a dependência da tecnologia estrangeira, visando mitigar os riscos do desequilíbrio da maturidade tecnológica resultantes de maquinário antigo e processos inadequados às condições mínimas necessárias para a transformação da indústria local com a velocidade necessária para o contexto 4.0 e da nova perspectiva de competitividade global.

A apresentação por parte da Federação de uma estratégia industrial com visão míope autocentrada de segredo industrial e a indefinição de uma dinâmica de incentivo à participação e engajamento dos atores por algo além da reatividade, torna incapaz a indústria Fluminense transcender a letargia da simples sobrevivência, incompatível com a lógica empresarial da economia pós-industrial. Para estudos futuros recomenda-se: (1) a realização de pesquisas envolvendo um número maior de empresas e de outros estados do Brasil; (2) a realização de estudos que explorem no médio e longo prazo a harmonização dos serviços e modelos empresariais por meio da introdução dos padrões internacionais de referência, como o alemão.

\section{Referências}

ADVANCED MANUFACTURING PARTNERSHIP (AMP): Institucional. Disponível em: https:// www.manufacturing.gov/. Acesso em: 26 ago. 2017.

ARTEMIS INDUSTRY ASSOCIATION: Institucional. Disponível em: https://artemis-ia.eu/about_ artemisia.html/. Acesso em: 27 ago. 2017.

BRAGA, J.; BRAGA, S. Ação empresarial em escala regional: um estudo da permeabilidade do Legislativo do Paraná à ação da Federação das Indústrias do Estado do Paraná (Fiep) na 16 legislatura (2007-2011). Revista Sociedade e Estado, v. 29, n.3, p. 895-919, 2014.

CONFEDERAÇÃO NACIONAL DA INDÚSTRIA: Estatuto CNI .10 Ofício de Registro de Pessoas Jurídicas do Distrito Federal sob o n0 00002248 do livro n0 A-03, protocolado e microfilmado sob o n0 00084150, Brasília abril de 2008.

CONFEDERAÇÃO NACIONAL DA INDÚSTRIA(CNI): Institucional. Disponível em: http://www. portaldaindustria.com.br/cni/institucional/conheca-cni. Acesso em: 25 ago. 2017.

CRESWELL. J. Projeto de pesquisa: métodos qualitativo, quantitativo e misto; tradução Luciana de Oliveira da Rocha. - 2. ed. - Porto Alegre: Artmed, 2007. 
DOCTOR, M. Business and delays in port: Reform in Brazil. Revista Brasileira de Economia Política, v. 22, n.2, p. 79-101, 2002.

DOSI, G. Technological paradigms and technological trajectories. Research Policy, v. 11, n.3, p.147-162, 1982.

FEDERAÇÃO DAS INDÚSTRIAS DO RIO DE JANEIRO (FIRJAN): Estatuto FIRJAN. Registrado no Cartório de Registro de Civil das Pessoas Jurídicas da Comarca do Rio de Janeiro de 2015.

FEDERAÇÃO DAS INDÚSTRIAS DO RIO DE JANEIRO (FIRJAN): Quem somos. Disponível em: http://www.firjan.com.br/o-sistema-firjan/setores-de-atuacao. Acesso em: 25 ago. 2017.

FREEMAN, C.; SOETE, L. The economics of industrial innovation. MIT Press, 1997.

FREEMAN, C. The 'National System of Innovation' in historical perspective. Cambridge Jour nal of Economics, v. 19, p. 5-24, 1995.

INDIAN INSTITUTE OF TECHNOLOGY HYDERABAD (IITH): Institucional Disponível em: https://www.iith.ac.in/index.php/initiatives//. Acesso em: 26 ago. 2017.

INSTITUTE OF MICROELECTRONICS OF THE CHINESE ACADEMY OF SCIENCES (IMECAS): Institucional Disponível em: http://english.ime.cas.cn/Research/ResearchDivisions/IoT1//. Acesso em: 27 ago. 2017.

JARDIM-GONÇALVES, R.; ROMERO, D.; GRILO, A. : Factories of the future: Challenges and leading innovations in intelligent manufacturing. International Journal of Computer Integrated Manufacturing, v.30, n.1, p. 4-14, 2017.

KAGERMANN, H.; WAHLSTER, W.; HELBIG, J. National academy of science and engineering: securing the future of German manufacturing industry. Recommendations for implementing the strategic initiative INDUSTRIE 4.0 Final report of the Industrie 4.0 Working Group, 2013.

LASTRES, H. M. M.; CASSIOLATO, J. E. Glossário de arranjos e sistemas produtivos e inovativos locais. Rio de Janeiro: SEBRAE, 2005. 
MAMEDE, M., et al. Sistema nacional de inovação: uma análise dos sistemas na Alemanha e no Brasil. Navus, v. 6, n. 4, p. 6-25, 2016.

MAPA DO DESENVOLVIMENTO DO ESTADO DO RIO DE JANEIRO: Disponível em: http:// www.firjan.com.br/o-sistema-firjan/mapa-do-desenvolvimento/. Acesso em: 27 ago. 2017.

MANCUSO, W. O empresariado como ator político no Brasil: Balanço da literatura e agenda de pesquisa. Revista de Sociologia e Política, v.28, p.131-146, 2007.

MAZZUCATO, M. Building the entrepreneurial state: A new framework for envi-sioning and evaluating a mission-oriented public sector, Working Paper. Levy Economics Institute, n. 824, 2015.

NELSON, R R. National Systems of Innovation: a comparative study. Oxford University Press,Oxford, 1993.

NETWORKING AND INFORMATION TECHNOLOGY RESEARCH AND DEVELOPMENT (NITRD): Institucional. Disponível em: https:// https://www.nitrd.gov//. Acesso em: 26 ago. 2017.

PINSKY, V.C.; MORETTI, S. L. A.; KRUGLIANSKAS, I.; PLONSKI, G. A. Inovação sustentável: Uma perspectiva comparada da literatura. Internacional e Nacional. Revista de Administração e Inovação, v.12, n. 3, p.226-250, 2015.

RELATÓRIO ANUAL DE INOVAÇÃO SENAI 2016: Disponível em: http://www.firjan.com.br/ publicacoes/publicacoes-de-inovacao/relatorio-anual-de-inovacao.htm. Acesso em: 20 ago. 2017.

RESELE, L. Impact of the national innovation system on innovation. Journal of Business Management, v. 9, p.52-62, 2015.

SOUZA, J. C.; FARIA, B.M.D.F. Processo de inovação no contexto organizacional: uma análise de facilitadores e dificultadores. Brazilian Business Review, v. 10, n.3, p. 113-136, 2013.

SOUZA, R.C. A inovação na micro e pequena empresa de software. Dissertação. 120f. 2016. (Mestrado profissional) - IBMEC, Rio de Janeiro, RJ, 2016.

WANG, S.; WAN, J.; LI, D.; ZHANG, C. (2016). Implementing smart factory of industrie 4.0: An outlook. International Journal of Distributed Sensor Networks, v.12, n.1, p. 1-12, 2016. 